\title{
The World Health Organization global aDSM database: generating evidence on the safety of new treatment regimens for drug-resistant tuberculosis
}

\author{
Christine M. Halleux ${ }^{1,3}$, Dennis Falzon ${ }^{2,3}$, Corinne Merle ${ }^{1}$, Ernesto Jaramillo², \\ Fuad Mirzayev², Piero Olliaro ${ }^{1}$ and Karin Weyer $^{2}$
}

Affiliations: ${ }^{1}$ Special Programme for Research and Training in Tropical Diseases (TDR), World Health Organization (WHO), Geneva, Switzerland. ${ }^{2}$ Global TB Programme (GTB), World Health Organization (WHO), Geneva, Switzerland. ${ }^{3}$ Both authors contributed equally.

Correspondence: Christine M. Halleux, Special Programme for Research and Training in Tropical Diseases (TDR), World Health Organization, Avenue Appia 20, 1211 Geneva 27, Switzerland. E-mail: halleuxclawho.int

@ERSpublications

The World Health Organization has created a central database to improve knowledge on drug-related harms of patients on novel tuberculosis regimens http://ow.ly/lfVm30ihRcO

Cite this article as: Halleux CM, Falzon D, Merle C, et al. The World Health Organization global aDSM database: generating evidence on the safety of new treatment regimens for drug-resistant tuberculosis. Eur Respir J 2018; 51: 1701643 [https://doi.org/10.1183/13993003.01643-2017].

Marketing new medicines before pre-registration clinical trials are complete may sound rash and risky. But when treatment options are scarce in the face of a lethal, transmissible disease the balance tilts in favour of "use" rather than "wait". Such were the conditions under which bedaquiline and delamanid (two new tuberculosis (TB) agents) were introduced a few years ago. This article relates how uncertainties about the safety of these new drugs were handled to enable their use.

Effective medications for TB strains resistant to isoniazid and rifampicin (multidrug-resistant (MDR)-TB strains) are scarce. The World Health Organization (WHO) estimates that 580000 new TB cases emerge each year requiring treatment for MDR-TB [1]. MDR-TB regimens in widespread use remain hugely unsatisfactory and only about one-half of patients globally complete treatment successfully, a statistic which has stagnated for several years. The long duration of the regimens and their toxicity are major determinants of unfavourable outcomes. Many patients who are not cured die or slip into chronicity and continue transmitting drug-resistant Mycobacterium tuberculosis strains to others [2, 3].

The arrival of bedaquiline in late 2012, the first of two new medicines targeted for MDR-TB, presented an opportunity to break this deadlock. After decades without new TB medicines, in recent years bedaquiline and delamanid received conditional approval for use in the treatment of MDR-TB by stringent drug regulatory authorities, including the European Medicines Agency and, in the case of bedaquiline, the US

Received: Aug 112017 | Accepted after revision: Jan 292018

The authors are staff members of the World Health Organization (WHO). They alone are responsible for the views expressed in this publication and they do not necessarily represent the decisions or policies of WHO. The designations used and the presentation of the material in this publication do not imply the expression of any opinion whatsoever on the part of WHO concerning the legal status of any country, territory, city or area, or of its authorities, nor concerning the delimitation of its frontiers or boundaries.

Conflict of interest: None declared.

The content of this work is copyright of the authors or their employers. Design and branding are copyright @ERS 2018. 
Food and Drug Administration [4-6]. WHO also issued interim policies on use of these two medicines as part of new MDR-TB and extensively drug-resistant (XDR)-TB regimens [7-9]. The incomplete safety profile of the two agents from phase IIB trial data was mitigated by advice to use them under conditions in which intensive monitoring for adverse events (AEs) could be done. The main safety concerns for bedaquiline included QT-interval prolongation and cardiac events, increase in hepatic transaminases and deaths; and for delamanid included QT-interval prolongation. However, these signals emerged from a limited number of patient observations and in most of the clinical studies, the safety data from cardiac monitoring provided only limited details, and therefore additional safety data are needed [7-15].

Unlike other monitoring activities inherent to TB programmes, such as sputum bacteriology, drug resistance and treatment outcomes, drug safety has not been consistently monitored by TB programmes in the past, raising concerns about the feasibility of implementing this requirement. This prompted WHO and other key stakeholders to define the parameters and essential steps needed for national TB programmes to establish an appropriate framework for what became known as "active TB drug safety monitoring and management" (aDSM) [16, 17] (table 1). aDSM has since then also been recommended for other novel TB treatment approaches for which safety data are sparse, such as the shorter MDR-TB regimen recommended by WHO since 2016 [3].

\section{aDSM: rationale and vision}

aDSM consists of the active and systematic clinical and laboratory assessment of patients on treatment with new TB medicines, or novel MDR-TB or XDR-TB regimens, to detect, manage and report suspected or confirmed drug toxicities. While all AEs detected need to be managed, the core package of aDSM requires that as a minimum, national programmes document and report all serious adverse events (SAEs) that occur in patients monitored. Data reported should include information on the SAE and drug exposures for all drugs taken by the patient experiencing the SAE. Treatment sites may also decide to include other AEs of special interest or clinical significance [16]. Whatever the level of monitoring, countries need to maintain databases containing the safety data obtained from aDSM. For this purpose they may create an electronic register, or accommodate the capture of additional variables in an existing electronic medical record system, following good practices in electronic recording [19, 20]. National aDSM databases do not intend to duplicate existing national collection of information on adverse drug reactions by the national pharmacovigilance centres, but rather seek to collect more detailed information or data on events that would have otherwise not been systematically collected.

Aside from a few high-burden settings, it is not expected that the volumes of patients recruited by any single country on aDSM would be large enough to permit meaningful analysis. Pooling data from different sites would thus be indispensable to better characterise the frequency of drug-related harms and increase

TABLE 1 Definitions of basic terms as applied in active tuberculosis (TB) drug safety monitoring and management (aDSM)

Term Definition

Adverse event

Serious adverse event

\section{Causal relationship}

\section{Adverse event of special interest}

\section{Adverse event of clinical significance}

Any untoward medical occurrence that may present in a TB patient during treatment with a pharmaceutical product, but which does not necessarily have a causal relationship with this treatment.

An adverse event which either leads to death or a life-threatening experience; to hospitalisation or prolongation of hospitalisation; to persistent or significant disability; or to a congenital anomaly. Serious adverse events that do not immediately result in one of these outcomes but that require an intervention to prevent it from happening are included [18]. Serious adverse events may require a drastic intervention, such as termination of the drug suspected of having caused the event.

A relationship between an exposure $(A)$ and an event $(B)$ in which $A$ precedes and causes $B$. This may refer to the causal association between an exposure to a TB medicine and the occurrence of an adverse reaction.

An adverse event documented to have occurred during clinical trials and for which the monitoring programme is specifically sensitised to report regardless of its seriousness, severity or causal relationship to the TB treatment [16].

An adverse event that either 1) is serious, 2) is of special interest, 3) leads to a discontinuation or change in the treatment, or 4 l is judged as otherwise clinically significant by the clinician [16]. 
the likelihood that uncommon ones are not missed. Involving the private sector would also be important in many countries. Starting in 2015, WHO's Global TB Programme (GTB) and Special Programme for Research and Training in Tropical Diseases (TDR) coordinated a dialogue with different stakeholders, which led to the creation of a global aDSM database to host national reports [17].

\section{The WHO global aDSM database}

The WHO global aDSM database collects a standard set of variables and comprises anonymised individual-level patient data on AEs (serious or not serious) from persons treated with new TB medicines or novel MDR-/XDR-TB regimens. Countries where aDSM has been implemented are invited to contribute by sharing their national data to the WHO global aDSM database. The data collected in the WHO global aDSM database cover demographic characteristics of the patient subject to the $\mathrm{AE}$, comorbidities, AE details (description of the event, start and stop date, intensity, seriousness, causal relationship, outcome and management), and information on all drug exposures at the time of the $\mathrm{AE}$ onset [21]. The causal link of an exposure with an AE is assessed at country level whenever possible (figure 1).

Country participation in the global aDSM database is governed by an agreement describing the responsibilities and the conditions of data use. Contributors retain ownership of their data and do not forfeit their ulterior use (e.g. research or publication). Participants are responsible for ensuring that national regulations are respected, especially regarding patient consent and data sharing, and including obtaining clearance from national ethical committees when applicable. Support for transfer, mapping and curation of the electronic dataset is provided [21]. Access to the aDSM global database is restricted to authorised staff designated by WHO for the purpose of data review and analysis. Reports of analyses are envisaged when sufficient data accrue, and should in the future generate additional evidence for treatment guidelines. Some high MDR-TB burden countries that are among the first to implement aDSM have shared their data and the expectation is that more countries will contribute to the aDSM global database in future. Data acquisition is slow, probably reflecting the relatively low pace of introduction of bedaquiline in most countries and the time it may take in some instances to formalise the data-sharing agreement, but usually contributing countries transfer data on a regular basis as they progress in enrolling patients under the new drugs and new regimens. Data obtained so far include repurposed drugs or bedaquiline, but no data have yet been collected in the aDSM global database on delamanid.

A number of challenges are anticipated. The success of the global aDSM database will depend upon the appreciation by treatment programmes of the importance of collecting and sharing data to improve knowledge on TB treatment. For this they need to invest time and resources to develop appropriate systems and implement aDSM. Until standardisation of methods improves, heterogeneity is expected between reporting sites, such as differing judgement on the drug-exposure relationship, quality and completeness of data, and the coding of AEs and medications [22-24]. A number of measures are being

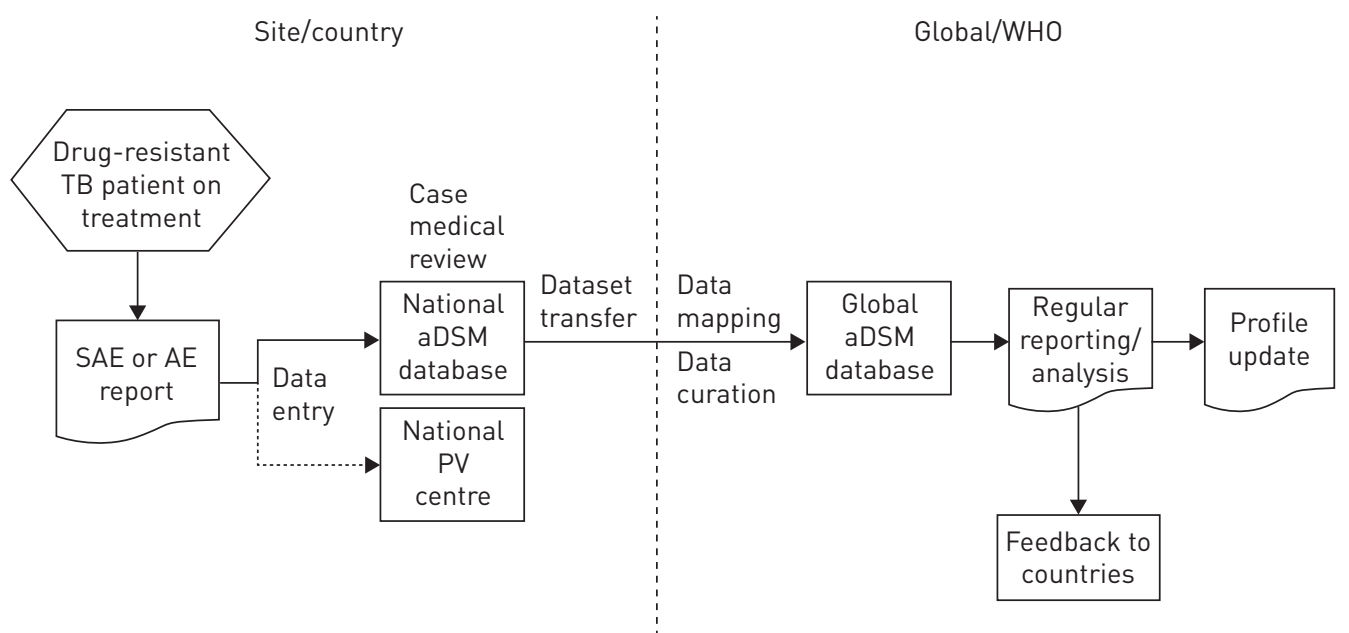

FIGURE 1 Schematic representation of the flow of data from the point of care to the global active tuberculosis (TB) drug safety monitoring and management (aDSM) database. Note that aDSM may collect more information than the regular pre-existing form for report of adverse drug reaction at country level. When the event ladverse event (AE) or serious adverse event (SAE)] qualifies for reporting to the national pharmacovigilance (PV) centre, national systems should be in place to ensure that the report is shared with the national PV centre. WHO: World Health Organization. 
taken to address these barriers. A dedicated website has been created to centralise information and training material to support contributors [17]. A special software (PViMS) has been developed by Management Sciences for Health (Arlington, VA, USA) to assist countries in capturing the necessary AE data with the consistency checks required to improve quality, validity and comparability of records [25]. Data sharing has not yet become standard practice and remains unfamiliar to many programmes [26]. However, advocacy for data sharing is increasing and its value in informing TB treatment policy is becoming clearer to many TB practitioners [27].

Once aDSM becomes a mainstream TB programme activity, it is expected that its value will extend beyond the individual patient monitored, to benefit other patients from improved knowledge of the medicines studied as well as endowing programmes with a robust mechanism to enable the introduction of future TB treatments at a swifter pace.

\section{Acknowledgements}

The authors wish to thank Michel Vaillant (Luxembourg Institute of Health) for technical support in setting up the global aDSM database, as well as the external experts who participated in the development of the framework (see [15], annex 1).

Author contributions: All authors contributed to the ideation and writing of this manuscript, and agree with the conclusions drawn.

\section{References}

1 World Health Organization. Global Tuberculosis Report 2016. WHO/HTM/TB/2016.13. Geneva, World Health Organization, 2016. http://apps.who.int/medicinedocs/documents/s23098en/s23098en.pdf

2 Bloss E, Kuksa L, Holtz TH, et al. Adverse events related to multidrug-resistant tuberculosis treatment, Latvia, 2000-2004. Int J Tuberc Lung Dis 2010; 14: 275-281.

3 Falzon D, Schünemann HJ, Harausz E, et al. World Health Organization treatment guidelines for drug-resistant tuberculosis, 2016 update. Eur Respir J 2017; 49: 1602308.

4 US Food and Drug Administration (FDA). Drug Approval Package. SIRTURO (bedaquiline) $100 \mathrm{mg}$ Tablets Company: Janssen Research and Development, LLC. Application No. 204384. www.accessdata.fda.gov/drugsatfda docs/nda/2012/204384Orig1s000TOC.cfm Approval date: December 28, 2012. Date last accessed: February 19, 2018.

5 European Medicines Agency. European public assessment report (EPAR) summary for the public. Deltyba (delamanid). London, European Medicines Agency, 2014. www.ema.europa.eu/docs/en_GB/document_library/ EPAR_-_Summary_for_the_public/human/002552/WC500166235.pdf

6 European Medicines Agency. European public assessment report (EPAR) summary for the public. Sirturo (bedaquiline). London, European Medicines Agency, 2014. www.ema.europa.eu/docs/en_GB/document_library/ EPAR___Summary_for_the_public/human/002614/WC500163211.pdf

7 World Health Organization. The use of bedaquiline in the treatment of multidrug-resistant tuberculosis. Interim policy guidance. WHO/HTM/TB/2013.6. Geneva, World Health Organization, 2013. http://apps.who.int/iris/ bitstream/10665/84879/1/9789241505482_eng.pdf

8 World Health Organization. The use of delamanid in the treatment of multidrug-resistant tuberculosis. Interim policy guidance. WHO/HTM/TB/2014.23. Geneva, World Health Organization, 2014. http://apps.who.int/iris/ bitstream/10665/137334/1/WHO_HTM_TB_2014.23_eng.pdf

9 World Health Organization. The use of delamanid in the treatment of multidrug-resistant tuberculosis in children and adolescents. Interim policy guidance. WHO/HTM/TB/2016.14. Geneva, World Health Organization, 2016. http://apps.who.int/iris/bitstream/10665/250614/1/9789241549899-eng.pdf

10 Pym AS, Diacon AH, Tang SJ, et al. Bedaquiline in the treatment of multidrug- and extensively drug-resistant tuberculosis. Eur Respir J 2016; 47: 564-574.

11 Migliori GB, Pontali E, Sotgiu G, et al. Combined use of delamanid and bedaquiline to treat multidrug-resistant and extensively drug-resistant tuberculosis: a systematic review. Int J Mol Sci 2017; 18: E341.

12 D'Ambrosio L, Centis R, Tiberi S, et al. Delamanid and bedaquiline to treat multidrug-resistant and extensively drug-resistant tuberculosis in children: a systematic review. J Thorac Dis 2017; 9: 2093-2101.

13 Matteelli A, D'Ambrosio L, Centis R, et al. Compassionate and optimum use of new tuberculosis drugs. Lancet Infect Dis 2015; 15: 1131-1132.

14 Gler MT, Skripconoka V, Sanchez-Garavito E, et al. Delamanid for multidrug-resistant pulmonary tuberculosis. N Engl J Med 2012; 366: 2151-2160.

15 World Health Organization. Active tuberculosis drug-safety monitoring and management (aDSM). Framework for implementation. WHO/HTM/TB/2015.28. Geneva, World Health Organization, 2015. http://apps.who.int/iris/ bitstream/10665/204465/1/WHO_HTM_TB_2015.28_eng.pdf

16 Pontali E, Sotgiu G, Tiberi S, et al. Cardiac safety of bedaquiline: a systematic and critical analysis of the evidence. Eur Respir J 2017; 50: 1701462.

17 World Health Organization. Active TB drug-safety monitoring and management (aDSM). www.who.int/tb/ areas-of-work/drug-resistant-tb/treatment/pharmacovigilance/en/ Date last accessed: June 22, 2017.

18 International Conference on Harmonisation of Technical Requirements for Registration of Pharmaceuticals for Human Use (ICH). ICH Harmonised Tripartite Guideline. Post-Approval Safety Data Management: Definitions and Standards for Expedited Reporting (E2D). Geneva, ICH, 2003. www.ich.org/fileadmin/Public_Web_Site/ICH_ Products/Guidelines/Efficacy/E2D/Step4/E2D_Guideline.pdf

19 Principles for Digital Development. http://digitalprinciples.org Date last accessed: June 22, 2017.

20 Timimi H, Falzon D, Glaziou P, et al. WHO guidance on electronic systems to manage data for tuberculosis care and control. J Am Med Inform Assoc 2012; 19: 939-941. 
21 World Health Organization. WHO global database for TB active drug safety monitoring home page. www.who. int/tdr/research/tb_hiv/adsm/en/ Date last accessed: June 22, 2017.

22 Medical Dictionary for Regulatory Activities (MedDRA). www.meddra.org Date last accessed: June 22, 2017.

23 Uppsala Monitoring Centre. What is WHO-ART? www.who-umc.org/vigibase/services/learn-more-about-who-art/ Date last updated: December 14, 2017. Date last accessed: February 19, 2018.

24 WHO Collaborating Centre for Drug Statistics Methodology. ATC/DDD Index. www.whocc.no/atc_ddd_index/ Date last accessed: February 19, 2018.

25 USAID, Systems for Improved Access to Pharmaceuticals and Services (SIAPS). Pharmacovigilance Monitoring System (PViMS). Arlington, Management Sciences for Health, 2015. http://apps.who.int/medicinedocs/documents/ s22248en/s22248en.pdf

26 Chatham House. Perspectives on Data Sharing in Disease Surveillance. London, The Royal Institute of International Affairs, 2014. www.chathamhouse.org/sites/files/chathamhouse/home/chatham/public_html/sites/ default/files/20140430DataSharingDiseaseSurveillanceRoss.pdf

27 Ahuja SD, Ashkin D, Avendano M, et al. Multidrug resistant pulmonary tuberculosis treatment regimens and patient outcomes: an individual patient data meta-analysis of 9,153 patients. PLoS Med 2012; 9: e1001300. 